\title{
Des modalités de l'énonciation en littérature orale
}

Jean Derive

\section{(2) OpenEdition}

Journals

Édition électronique

URL : https://journals.openedition.org/clo/1127

DOI : $10.4000 /$ clo. 1127

ISSN : 2266-1816

Éditeur

INALCO

\section{Édition imprimée}

Date de publication : 1 janvier 2009

Pagination : $91-110$

ISBN : $978-2-85831-182-8$

ISSN : 0396-891X

\section{Référence électronique}

Jean Derive, "Des modalités de l'énonciation en littérature orale », Cahiers de littérature orale [En ligne], 65 | 2009, mis en ligne le 01 mars 2013, consulté le 30 juin 2021. URL : http://

journals.openedition.org/clo/1127 ; DOI : https://doi.org/10.4000/clo.1127

Ce document a été généré automatiquement le 30 juin 2021.

\section{(c) (†) 8)}

Cahiers de littérature orale est mis à disposition selon les termes de la Licence Creative Commons Attribution - Pas d'Utilisation Commerciale 4.0 International. 


\title{
Des modalités de l'énonciation en littérature orale
}

\author{
Jean Derive
}

1 La littérature n'est pas simplement la version écrite de l'oraliture, comme si le passage de l'un à l'autre mode ne modifiait en rien les conditions d'énonciation du discours. Dans les faits, tant du point des modalités matérielles de cette énonciation que des sujets qui la prennent en charge, les processus à l'œuvre sont très différents.

\section{Les modalités matérielles de l'énonciation en littérature orale}

2 En régime d'oralité littéraire - d'oralité première en tout $\operatorname{cas}^{1}$ - les modalités de l'énonciation supposent, à la différence de ce qui se passe pour la littérature, la présence hic et nunc des partenaires de la communication. Cette particularité a des incidences sur les facteurs de l'énonciation qui sont soumis à une double contrainte :

- du point de vue du temps, les discours de l'oralité première ne peuvent être consommés que dans l'immédiateté de leur énonciation, alors qu'au contraire, la lecture peut être différée et fragmentée à loisir. En outre, beaucoup de discours patrimoniaux ne peuvent s'énoncer qu'en des temps prescrits (moments de la journée, saison, ou dates calendaires...), à la différence encore de la lecture qui, dans la majorité des cas pour ce qui concerne la littérature, relève du bon plaisir du consommateur ;

- du point de vue de l'espace, ces mêmes discours patrimoniaux doivent également se dire dans des espaces prévus à cet effet et selon des configurations précises. Certes, dans le cas de la littérature, la mise en espace du texte n'est pas non plus indifférente. Selon qu'il figure sur un rouleau, sur un livre... ce texte peut déjà prendre une connotation différente. Son édition n'aura pas non plus la même signification selon que le volume est in quarto ou in octavo, selon la taille et le type des caractères choisis, selon la marge et l'interligne qui font que la page est plus ou moins remplie. Tous ces choix, qui sont des choix de mise en espace, orientent déjà la nature de l'énoncé avant même que la lecture soit commencée : texte poétique ou texte ludique (plus aérés) ou essai savant (occupation plus dense de l'espace), 
etc. Mais en littérature, la lecture peut néanmoins se faire en n'importe quel lieu et par conséquent le déterminisme du critère spatial semble infiniment moins contraignant.

3 Nous développerons ici le paramètre de l'espace de la performance, plus complexe et moins étudié que celui du temps. Nous l'illustrerons à partir du cas de la littérature orale mandingue appartenant à une aire culturelle importante d'Afrique de l'Ouest. Au Manding, à l'instar de ce qui se passe dans la plupart des cultures orales, il n'est pas permis d'énoncer les principaux genres du patrimoine oral n'importe où. Les lieux où ils doivent être proférés sont réglementés. Il peut même exister des interdits « locatifs » qui excluent qu'ils soient exécutés en dehors d'espaces prescrits.

\section{Le lieu de production des genres à partir de l'exemple manding}

4 Dans la civilisation mandingue, on peut distinguer trois grands types d'espace où se produisent les genres oraux : un espace public, un espace privé et un espace contingent, c'est-à-dire un espace qui est associé à une activité particulière à laquelle le genre oral se trouve intrinsèquement lié.

\section{L'espace privé}

5 Il sera défini comme celui de la cour familiale (lù), c'est-à-dire l'espace ménagé entre quelques maisons d'habitation qui abritent une famille étendue dont le noyau est un foyer avec un chef de famille. C'est dans cet espace que les femmes pilent, cuisinent, mais c'est là aussi qu'on se réunira le soir, en saison sèche, pour dire en famille des contes ou des devinettes, soirées auxquelles pourront facilement s'adjoindre quelques amis ou voisins. À la cour peut aussi bien se substituer la pièce intérieure d'une habitation qui la borde, si l'auditoire est restreint ou si les conditions météorologiques l'exigent. C'est sur ce même espace privé que pourront être exécutés les chants attachés à quelque cérémonie concernant un membre de la famille, telle que baptême, mariage ou funérailles, à condition que les participants ne soient pas trop nombreux. Lorsqu'il s'agit en effet d'une famille de notables, souvent numériquement importante, ces mêmes cérémonies et les œuvres de littérature orale qui les accompagnent se dérouleront alors plutôt sur un espace public, à l'échelle du quartier (kàbila) regroupant un ensemble cohérent de lignages, ou encore à l'échelle de l'agglomération entière, suivant sa taille.

6 En revanche, les chroniques historiques ayant trait à l'origine des familles et à leurs migrations doivent impérativement se dire dans l'espace privé, cela semble-t-il afin d'éviter les confrontations entre les différentes chroniques familiales qui seraient susceptibles de faire apparaitre des contradictions et par conséquent de provoquer des querelles entre familles. Seules des chroniques concernant un ensemble important de lignages ou l'ensemble de l'agglomération seront dites sur un espace public, à l'échelle du kàbila ou du village.

\section{L'espace public}

7 Pour un certain nombre de manifestations culturelles, dont les performances de plusieurs genres de littérature orale, est prévu au Manding un espace cérémoniel à caractère public appelé fèrè qui peut exister aussi bien à l'échelle du quartier que de l'ensemble de l'agglomération si elle est de moindre importance. C'est là que se 
déroulent la plupart des chants accompagnant les danses, là aussi que sont interprétées les épopées (fàsa), là que se déroulent les chants de cérémonie impliquant la collectivité au-delà de l'intimité d'une famille particulière. Ainsi les chants attachés à certaines fêtes islamiques sont-ils traditionnellement dits sur l'espace public, de même par exemple que les chants de circoncision ou d'excision, dans la mesure où ces rituels sont la plupart du temps collectifs et que c'est toute une classe d'âge qu'on circoncit ou qu'on excise.

\section{L'espace contingent}

8 Certains genres de la littérature orale mandingue sont intrinsèquement liés à des occupations affectées à un espace spécifique et bien entendu les œuvres de ces répertoires sont exécutées sur le lieu où se déroule cette activité. Ainsi, plusieurs catégories de chants agricoles sont-elles dites aux champs à l'occasion de certaines pratiques agricoles (semailles, sarclage...), espace où l'on retrouvera d'ailleurs la possibilité d'organiser des séances de contes et de devinettes, lorsqu'en saison d'activité intense on reste dormir dans des huttes champêtres aux abords des terres cultivées. De même, plusieurs types de chants ou de récits de chasseurs sont-ils interprétés en brousse, en des lieux ritualisés (notamment certains carrefours), tandis que d'autres peuvent aussi être exécutés sur l'espace public.

9 Il arrive également que la production de certains genres soit liée à un déplacement dans l'espace et qu'elle corresponde par conséquent à un itinéraire plutôt qu'à un lieu fixe et bien circonscrit. C'est le cas par exemple des chants de masques des Dioula de Kong, en Côte-d'Ivoire, qui sont interprétés au fur et à mesure d'un parcours préétabli suivi par le masque à l'intérieur de l'agglomération. Au Manding, certains chants de mariage, comme ceux où la mariée fait ses adieux rituels aux différents membres de sa famille (puisqu'elle va devoir intégrer celle de son mari), relèvent eux aussi de ce type de fonctionnement spatial, dans la mesure où la mariée accomplit un itinéraire.

Ces différents exemples font bien apparaître qu'au Manding, la parole littéraire est bien contrôlée dans l'espace (comme elle l'est d'ailleurs dans le temps) et que n'importe quel énoncé ne peut pas se dire n'importe où. Outre la spécification du lieu de production du discours, le critère spatial intervient encore d'une autre manière pour la détermination des genres, dans la mesure où les performances des divers genres s'organisent différemment de ce point de vue.

\section{La mise en espace de la performance}

11 À ce propos, on rencontre plusieurs cas de figure dont nous allons, à titre indicatif, indiquer les plus courants :

12 Cas no 1 : l'interprète d'une part et l'auditoire d'autre part sont stables tout au long de la performance et nettement définis l'un par rapport à l'autre, l'exécutant (et d'éventuels acolytes) se trouvant situé(s) à l'extérieur de l'espace occupé par l'assistance. C'est ainsi qu'au Manding s'organise par exemple la performance de l'épopée (fàsa) où l'on trouvera d'un côté le griot (jèli) et son musicien répondant et de l'autre les auditeurs-spectateurs.

13 Cas $\mathbf{n}^{\circ} \mathbf{2}$ : l'interprète et l'auditoire sont toujours clairement définis l'un par rapport à l'autre, mais le premier est cette fois entouré de l'assistance qui délimite un espace clos 
autour de lui. Selon la nature des genres exécutés, il peut être ou non mobile à l'intérieur de cet espace où il est enfermé. Quelquefois, l'exécution de l'épopée peut aussi connaître cette configuration et, dans ce cas, l'interprète ne se déplace pas au cours de sa performance. En revanche, dans le cas de l'exécution du bàra (pot-pourri chanté et dansé par un spécialiste accompagné d'un musicien), l'énonciateur se déplace tout au long de la performance à l'intérieur de l'espace circonscrit.

Cas $\mathbf{n}^{\circ} 3$ : l'auditoire délimite un espace clos (circulaire, ovoïde ou quadrilatéral) comme dans l'exemple précédent, mais la performance connaît une succession d'interprètes qui prennent alternativement la parole depuis l'endroit qu'ils occupent dans l'assistance. C'est ce qu'on rencontre dans les séances de contes ou de devinettes.

Cas $\mathbf{n}^{\circ} 4$ : il s'agit d'une organisation spatiale assez proche de la précédente à cette différence près que les interprètes successifs viennent à tour de rôle au centre de l'espace clos. Ainsi fonctionnent au Manding beaucoup de chants de danse des femmes qui viennent alternativement (le plus souvent par duo) chanter et danser des pièces du répertoire.

Cas $\mathbf{n}^{\circ} 5$ : la performance donne lieu tout au long à une énonciation collective et il n'est donc pas nécessaire de distinguer l'espace des interprètes de l'espace de l'assistance. En général, dans ce cas-là, les participants se regroupent en cercle et tous chantent depuis le pourtour de la circonférence. Cette mise en espace est adoptée pour l'exécution de toute une série de chants, notamment à l'occasion de cérémonies nuptiales.

17 Cas $\mathbf{n}^{\circ} \mathbf{6}$ : les participants à la séance occupent dans l'espace deux groupes distincts qui peuvent selon les genres prendre la forme de deux cercles ou de deux rangs se faisant face. Cette configuration est adoptée pour des chants agonistiques où les deux groupes (en général des hommes et des femmes) entonnent alternativement des chants pour se railler réciproquement.

18 Ces différentes mises en espace ne sont évidemment pas des mises en scène arbitraires. Elles définissent chaque fois un type de relation performateur/auditoire selon par exemple que l'énonciateur est ou non un spécialiste, selon que l'on considère la performance comme un spectacle à consommer ou bien comme un rituel collectif où chacun peut être effectivement ou potentiellement acteur. Le critère de l'espace, tant par la prescription des lieux que par la configuration spatiale de la séance, peut donc être parmi d'autres un facteur opératoire pour penser une typologie des genres de la littérature orale, comme l'a montré l'exemple manding. Il est clair en effet que si le critère de la mise en espace peut fonctionner en littérature comme en oraliture, il a un rôle beaucoup plus important dans ce second régime pour déterminer la nature des genres littéraires.

19 À propos de la comparaison contrastive des modalités respectives de l'énonciation en littérature et en oraliture, un autre point capital concerne la question du sujet de l'énonciation.

\section{Le sujet de l'énonciation en littérature orale}

20 En général, dans la tradition de la réception de la littérature écrite, le sujet de l'énonciation d'une "œuvre", offerte par la lecture à la consommation d'un public, c'est d'abord un auteur nommé et par conséquent connu sur la couverture du livre. Il est supposé être "l'inventeur» du discours proposé, c'est-à-dire une conscience 
individuelle à l'origine d'une création "maîtrisée ", même si depuis le $\mathrm{xx}^{\mathrm{e}}$ siècle, la réalité de ce sujet est théoriquement relativisée comme source de la création :

- d'une part, parce que certains courants, comme le surréalisme en Europe avec l'écriture automatique, ont milité pour une évacuation maximale du sujet conscient, préférant lui substituer le « hasard objectif»;

- d'autre part, parce que les sciences humaines, notamment la psychanalyse et la sociologie, ont mis en évidence que, de toute façon, ce sujet était toujours débordé par son propre acte créateur. La psychanalyse nous apprend que, dans son énoncé, il inscrit du sens à son insu et même parfois contre son gré (mais c'est encore le sujet, même s'il n'est plus tout à fait conscient). En outre, ajoute la sociologie, ce qu'il croit dire de son propre chef est en réalité dicté par une série de déterminismes qui pèsent sur lui : il est lui-même le produit d'un habitus qui informe son discours, si bien que la conscience individuelle n'est plus la source unique du sens.

21 Cela dit, la figure du sujet de l'énonciation du discours littéraire représentée par l'auteur n'est pas la seule à intervenir pour façonner le produit qui, en fin de compte, sera celui qui sera accessible à un vaste public. L'acte d'énonciation de l'auteur aboutit concrètement à un manuscrit ou plutôt à un "tapuscrit " comme on dit aujourd'hui, mais cette première performance énonciative n'est pas la performance définitive qui donnera lieu à la consommation culturelle généralisée du discours énoncé. Pour cela, un autre sujet intervient secondairement comme médiateur dans le processus d'énonciation, c'est l'éditeur, lui aussi bien visible sur l'objet-livre, dont la fabrication correspond à l'ultime performance énonciative du discours littéraire. Son rôle est loin d'être négligeable. D'une part, il a des exigences qui peuvent amener l'auteur à revoir son texte en en corrigeant certains aspects tant du point linguistique que morphologique; d'autre part, il intervient dans les modalités d'énonciation, par le format, la couverture, la mise en page, etc.

Dans le domaine littéraire, il peut y avoir enfin un troisième sujet, virtuel, de l'énonciation, c'est l'énonciateur textuel, celui qui, en apparence, est censé prendre en charge l'énonciation. Le cas le plus connu est celui du récit où souvent un narrateur virtuel masque l'auteur, sujet réel de l'énonciation.

On pourrait être tenté de créer un parallèle avec la littérature orale en proposant des équivalences terme à terme. Aux œuvres orales, il y a bien eu un «inventeur » qu'on peut assimiler à l'auteur. L'interprète, qui réalise ces œuvres en une gamme d'énonciations particulières, serait quant à lui plus ou moins l'équivalent de l'éditeur, d'autant qu'en régime de scripturalité aussi une œuvre littéraire peut faire l'objet de plusieurs éditions par des éditeurs différents qui lui donneront chaque fois une physionomie un peu particulière. Quant à la mise en scène d'un énonciateur textuel fictif, elle est possible et attestée dans un cas comme dans l'autre. Les rituels de bien des contes oraux où, à la fin, le conteur s'affirme témoin et acteur de l'histoire qu'il a racontée l'illustrent parfaitement ${ }^{2}$. Il ne faut donc point confondre, en régime d'oralité non plus, le «je » de l'énonciateur textuel avec le «je » du performateur réel de l'énoncé qui nous est donné à entendre.

Pourtant, cette mise en parallèle ne fonctionne pas vraiment, car entre la scripturalité et l'oralité les situations et donc les processus ne sont pas les mêmes. Sous le régime de l'oralité, l'essentiel de ce qui est énoncé apparaît en effet comme l'expression d'une propriété verbale collective consignée dans un répertoire préexistant au sujet qui lui donne vie à l'occasion d'une performance donnée. Dans la grande majorité des cas la 
mémoire de l'inventeur (l'auteur) de l'œuvre a été perdue : ainsi en va-t-il dans toutes les cultures de la plupart des contes, chants populaires, histoires drôles, maximes et même de beaucoup de récits épiques.

Alors qu'en régime scriptural, la littérature, parce qu'elle peut conserver matériellement ses discours, permet d'instaurer une mémoire objective, ce qui la conduit à valoriser d'abord l'auteur comme sujet (on retient Homère, Shakespeare, Victor Hugo, plus que leurs éditeurs), le régime oral tend à l'effacement de l'inventeur comme sujet individuel au profit d'un sujet collectif. Cela ne veut évidemment pas dire qu'il n'y a pas eu d'inventeur. Mais, comme l'ont fait judicieusement remarquer R. Jakobson et P. Bogatyrev (1973, 59-72), cela tient au fait qu'en oralité, l'énonciation originelle n'ayant jamais été conservée et le discours ayant été remodelé par tant d'énonciations successives, il est devenu dans la conscience sociale un objet culturel effectivement communautaire que la société s'est progressivement approprié.

En oralité, le sujet principal de l'énonciation est l'interprète du discours qui l'actualise à un moment donné, ne serait-ce que parce qu'il est le seul concrètement visible au moment de cette énonciation. Il est présent hic et nunc pour son auditoire, à la différence d'ailleurs de l'auteur et de l'éditeur pour un lecteur. Pour cet auditoire, il est donc beaucoup plus important que l'éditeur d'un livre dans la mesure où il prend en charge de façon sensible la totalité du discours.

Il convient toutefois de s'interroger sur le statut de l'interprète comme sujet de l'énonciation. Est-il pour la collectivité qui l'écoute véritablement sujet ou simple instrument, selon quoi, dans cette seconde hypothèse, il serait plutôt l'équivalent du livre que de l'éditeur? La réponse à cette question est assez délicate, car il faut distinguer la représentation des usagers et la réalité de ce qui se passe, ces deux niveaux étant loin de coïncider.

Ce qu'attend la communauté de l'interprète de littérature orale, en régime d'oralité première en tout cas $^{3}$, est moins qu'il se manifeste en tant que personnalité individuelle originale et novatrice que comme héraut conforme d'une conscience collective. Dans la plupart des cultures orales, on ne demande pas à l'interprète d'innover, mais d'être fidèle à une tradition qui concernera aussi bien l'énoncé que l'énonciation. Selon ce mode de représentation, l'interprète n'est donc accepté comme sujet qu'en tant qu'il est apte à se faire l'écho de ce qui est supposé être une vision communautaire. À ce niveau encore, il y a donc un certain effacement du sujet comme individualité propre. Cet effacement est dans certains cas renforcé par le fait qu'en régime d'oralité, il peut y avoir coénonciation simultanée: beaucoup de chants par exemple peuvent s'interpréter en chœur, ce qui renforce le caractère collectif du sujet de l'énonciation et gomme les individualités. La coénonciation simultanée n'est certes pas ignorée de la littérature (livres écrits à deux voix), mais elle est beaucoup plus rare et elle reste le plus souvent l'addition de deux individualités plutôt que l'expression d'un point de vue communautaire qui parle d'une seule voix.

Est-ce à dire qu'en oralité l'artiste verbal ne serait jamais un créateur? Un tel point de vue, mythique, est contredit par la réalité de la variabilité des interprétations successives des œuvres qui, maintenant qu'on a pu enregistrer, pour certaines d'entre elles, un nombre important de performances, montre que l'interprète s'inscrit toujours dans son énoncé (même s'il n'est pas textuellement visible), lui faisant subir un traitement susceptible d'avoir de fortes incidences sur son sens et sur ses fonctions. Beaucoup d'études ont ainsi mis en évidence qu'un même conte pouvait prendre une 
physionomie très différente selon qu'il était dit, par exemple, par un homme ou par une femme, ou bien encore, en diachronie, par des interprètes de générations différentes. Mais avec ces illustrations, nous sommes encore dans le cas de sujets collectifs représentant non plus la totalité de la communauté, mais un groupe significatif de celle-ci. En oralité, l'acte créateur individuel du sujet de l'énonciation se réalise donc essentiellement dans les bornes d'un cadre dont les références collectives doivent rester sensibles.

Mais, bien entendu, l'interprète ne reproduit pas comme un magnétophone et l'énonciation orale d'œuvres patrimoniales étant fondée sur la mémoire, il y a toujours une part de subjectivité qui conduit ce dernier à adapter son discours en fonction de la sensibilité et de l'idéologie du moment. Les médiévistes français ont ainsi bien mis en évidence que le manuscrit d'Oxford de La Chanson de Roland ( $\mathrm{xl}^{\mathrm{e}}$ siècle), trace emblématique des performances que les jongleurs pouvaient réciter à l'époque, orientait cet épisode historique selon une problématique qui était celle des croisades (événements appartenant au temps de l'énonciation), plutôt que celle de l'époque des faits relatés (le désastre de Roncevaux au VIII ${ }^{\mathrm{e}}$ siècle). Par la tradition orale, l'Histoire, loin de se contenter de perpétuer un souvenir (le fameux «lieu de mémoire » cher à Pierre Nora), refonde donc perpétuellement la mémoire du passé communautaire par une relecture permanente de ce passé en fonction des valeurs du moment. C'est même paradoxalement grâce à cette variabilité que la tradition, qui suppose en théorie la conformité à un état antérieur, peut se perpétuer. C'est elle qui lui permet de s'adapter à l'évolution des valeurs, sinon elle deviendrait rapidement obsolète.

31 Examinons rapidement quelles peuvent être les modalités de l'inscription du sujet dans son énoncé au cours de l'acte d'énonciation en régime oral.

\section{Action de l'énonciateur sur son énoncé indépendamment d'éventuelles marques textuelles de sa présence}

\section{Actualisation contextuelle de l'énoncé}

32 La première opportunité qui s'offre à un interprète de littérature orale de se manifester comme sujet d'énonciation tient d'abord à l'opération sélective à laquelle il est contraint et qui sera révélatrice de sa personnalité individuelle et sociale. L'interprète en effet ne va pas tout énoncer du patrimoine verbal de sa communauté et il ne va même pas tout reprendre du répertoire communautaire d'un genre, se créant, au sein de celui-ci, son propre répertoire par des choix restrictifs. Cette sélection qu'il est amené à faire le manifeste déjà comme un sujet d'énonciation (Baumgardt, 2000) ayant un rôle actif.

Une deuxième occasion lui est offerte d'agir comme sujet de l'énonciation par la contextualisation particulière qu'il peut faire de son énoncé. Même s'il ne touche pas à son signifié tel qu'il se trouve consigné dans le patrimoine - modèle archétypal qui peut avoir une forme linguistique relativement stable dans le cadre d'énoncés brefs (les proverbes : "pierre qui roule n'amasse pas mousse ») ou très contraints (les chants, tributaires de leur mélodie) -, il peut orienter différemment son sens (à distinguer du signifié) en fonction d'un contexte particulier. Ainsi, le proverbe évoqué ci- dessus peut se dire à propos de quelqu'un que son instabilité ou sa dispersion empêche soit d'amasser des biens matériels, soit des biens intellectuels (à trop se disperser, on 
n'acquiert pas de diplôme), soit des relations interpersonnelles, etc. Cet énoncé peut par ailleurs s'entendre, suivant les contextes, soit comme un conseil, soit une mise en garde, soit comme une leçon donnée à quelqu'un après coup, soit enfin comme un commentaire adressé à un interlocuteur à propos d'un tiers... ce qui correspond à différentes orientations données par le sujet de l'énonciation. Dans le même ordre d'idées, en Afrique, par exemple, certains griots peuvent utiliser des proverbes ou des chants empruntés aux répertoires patrimoniaux pour faire la promotion d'un personnage important tel qu'un homme politique. Dans d'autres contextes, ces mêmes interprètes mettront ces énoncés au service d'une opération d'éducation pour le développement ou la santé.

Ce principe, très évident pour le genre parémique, peut être étendu à toute la littérature de circonstance, dont il est généralement assez facile de déplacer le sens en l'appliquant à des contextes différents qui recyclent en quelque sorte la portée du discours. C'est bien chaque fois l'énonciateur qui, par la mise en relation avec un contexte spécifique d'un énoncé dont il n'est pas l'inventeur, s'affirme comme sujet en choisissant de lui donner une orientation particulière.

Cette question de l'actualisation contextuelle se pose également dans le cas des coénonciations non plus cette fois simultanées, mais plutôt successives ou alternatives. Les cas de coénonciations successives se rencontrent surtout dans les genres qui s'énoncent dans le cadre de séances où il est d'usage d'enfiler les œuvres en série et où par conséquent se succèdent les interprètes; ainsi en va-t-il un peu partout pour les contes, les devinettes, les chants cérémoniels... Il est alors possible de considérer la séance comme une sorte d'acte de création collective. Dans sa globalité, elle prend en effet un sens spécifique déterminé par la combinaison particulière des œuvres qui y ont été produites. Dans un tel cas de figure, chaque sujet- interprète participe d'une certaine façon à la création, dans la manière qu'il a de se situer par rapport aux autres en choisissant, dans le chapelet des œuvres déroulées, la place et le moment de son intervention.

Les cas de coénonciation alternative quant à eux correspondent à certains genres qui sont exécutés par un interprète accompagné par un acolyte ou un chœur, ce qui est assez fréquent en Afrique, par exemple, jusque dans le genre conte où le narrateur principal est souvent assisté d'un «répondeur» qu'on appelle aussi «agent rythmique », qui scande et parfois commente les différentes séquences narratives ». On rencontre aussi cette coénonciation alternative dans tous les genres qui se présentent sous forme de joutes oratoires (entre deux individus, entre des groupes tels que clans, classes d'âge, groupes sexués) telles qu'on en retrouve en bien des civilisations. Enfin, dans la mesure où de nombreux genres oraux - notamment les contes - laissent à l'auditoire la possibilité d'intervenir ponctuellement par un commentaire, une question, voire simplement par des rires ou des applaudissements, mettant en œuvre une certaine interactivité, on peut encore considérer qu'il y a là une forme basique de coénonciation, impensable dans le cas de la lecture d'un livre. Toutes ces formes de coénonciation tendent encore à la dilution du sujet considéré comme individualité et correspondent à une situation différente de celle de la littérature écrite où l'acte créateur du sujet écrivant est en principe solitaire. 


\section{Traitement subjectif de l'œuvre par le sujet-énonciateur} en particulier les œuvres narratives, telles que contes, mythes ou épopées que les variantes de l'énoncé, figuratives ou fonctionnelles, sont les plus importantes et les plus significatives. Le conte, comme Propp l'avait bien aperçu, peut ainsi habiller une même fonction narrative par différentes figures : le héros à qui l'on a confié la tâche impossible de trier des graines peut se faire aider par un animal secourable qui, selon les versions, pourra être un oiseau, un rat, une fourmi, etc. On peut aussi rencontrer des variantes de l'énoncé qui sont quant à elles d'ordre fonctionnel: dans certaines versions du «Petit chaperon rouge », l'héroïne est dévorée par le loup, dans d'autres elle réussit à se sauver. Il est évident que cette fin «heureuse » ou «malheureuse » du conte en modifie considérablement la perspective, même si la morale n'en est pas fondamentalement changée, y compris dans sa dimension symbolique relative à la sexualité des jeunes filles.

Tous les chercheurs qui ont analysé un peu finement le phénomène de la variabilité du conte en fonction des énonciateurs ont fait la même constatation: loin d'être arbitraires, les variantes propres à chacun d'eux correspondent le plus souvent à des traitements cohérents de cette œuvre, dépendant de la «lecture » qui en est faite par chacun en fonction de la place (réelle ou imaginaire) qu'il s'assigne au sein de la société (Baumgardt, 2000). 


\section{Les variations de l'énonciation}

43

$$
\begin{aligned}
& \text { imprime aussi sa marque par la spécificité de son énonciation. Même dans le cas limite } \\
& \text { des textes appris par cœur et repris linguistiquement à l'identique, l'énonciation ne } \\
& \text { sera jamais tout à fait la même d'un interprète à un autre : la prosodie, la diction, la } \\
& \text { gestuelle imposeront toujours la réalité d'un sujet physiquement présent au moment de } \\
& \text { la performance, à la différence de ce qui se passe quand on consomme une œuvre } \\
& \text { écrite. Cette dimension de la littérature orale, qui disparaît lorsqu'elle a été transcrite, } \\
& \text { a été elle aussi bien étudiée, notamment à propos de l'Afrique subsaharienne (Finnegan, } \\
& 1967 \text {; Derive, 1975). }
\end{aligned}
$$

\section{Création de compositions originales}

Ce phénomène propre au régime d'oralité qui permet à chaque sujet énonciateur de créer des compositions originales dans le cadre de répertoires existants se rencontre en particulier dans le genre du conte. On sait depuis les travaux de l'école finnoise que les contes sont en partie composés de motifs plus ou moins interchangeables, qu'en tout cas on retrouve fréquemment d'un conte à un autre, au point que Stith Thompson a pu leur consacrer un index. Ce n'est pas le propos ici d'en faire le catalogue par ailleurs bien connu. Il s'agira simplement de constater que, sans créer d'œuvres absolument nouvelles, le conteur a la latitude de broder sur des canevas connus en donnant à ses contes par une combinaison personnelle de ces motifs préexistant à l'œuvre, une physionomie originale.

Ce procédé est nettement plus développé dans les communautés où l'oralité première, toujours bien vivante, représente une modalité dominante en matière de culture verbale, comme c'est le cas, entre autres, de la plupart des cultures africaines. Dans ces cultures, le phénomène évoqué se retrouve en de multiples genres, notamment dans les répertoires chantés. Dans ces répertoires, les véritables unités de base ne sont pas toujours des œuvres déjà toutes constituées, mais des unités discursives plus petites, ayant si l'on veut la fonction des pièces d'un jeu de construction. C'est l'assemblage de ces différentes pièces qui permet à chaque énonciateur individuel de construire, dans le cadre des règles d'un genre donné, une grande variété d'œuvres originales, grâce à un jeu infini de combinaisons possibles (Koudjo, 1989 ; Luneau, 1974). Le propos demande cependant à être nuancé, car si les combinaisons sont potentiellement infinies, dans la réalité, certains types d'assemblages ont tendance à s'imposer plus que d'autres et à créer effectivement des archétypes d'œuvres. La possibilité de telles combinaisons est donc encore un espace offert à l'énonciateur de littérature orale pour se manifester comme sujet.

\section{Création d'œuvres nouvelles}

Il ne faut pas oublier enfin que, même si ce cas de figure est minoritaire dans les productions de littérature orale, peuvent être également créées par certains interprètes des œuvres entièrement originales qui viennent enrichir les répertoires de différents genres. Ainsi en va-t-il un peu partout des histoires drôles par exemple. En beaucoup de cultures, des chansonniers collent aussi à l'actualité, notamment politique, et créent des chansons populaires qui, par un phénomène de bouche à oreille, 
sont bientôt sur toutes les lèvres de la communauté. Il est vrai que ce phénomène de créations nouvelles par des interprètes individuels est surtout répandu dans les situations de néooralité urbaine, qui souvent relèvent davantage de l'oralité seconde que de l'oralité première, car les œuvres, même si elles sont à l'origine improvisées oralement, sont souvent médiatisées par des supports individuels et peuvent par conséquent être matériellement stockées. En conséquence, le fait que ces répertoires de néooralité soient objectivement consultables (à la différence des œuvres produites en régime d'oralité première qui n'ont pas de réalité en dehors des performances) libère les interprètes de l'exigence de fidélité à un répertoire sans existence matérielle et les rend plus disponibles pour l'innovation et l'invention.

Mais la création d'œuvres nouvelles, pour être moins répandue dans des situations d'oralité première, n'est cependant pas absente dans cette configuration, comme en témoigne, une fois de plus, l'exemple des sociétés d'Afrique subsaharienne. Ainsi les pasteurs peuls, au retour de longs périples de nomadisation, composent-ils individuellement de longs poèmes originaux sur les robes de leurs vaches et d'ailleurs, la communauté attribue à chacun la paternité de ses textes (Seydou, 1991). Cette reconnaissance des auteurs est fréquente aussi pour les productions d'artistes spécialisés et salués comme tels. Par exemple les poètes musiciens nzakara (République centrafricaine) donnent souvent des chansons qu'ils ont inventées eux- mêmes (de Dampierre, 1963). Les plus talentueux d'entre eux peuvent même avoir tout un répertoire à leur actif (de Dampierre, 1987). Évidemment, ces créations ne sont reconnues par la communauté que dans la mesure où elles sont bien conformes aux normes traditionnelles du genre dans lequel elles s'inscrivent, puisque l'oralité première recommande la mimesis et proscrit l'innovation.

\section{Les marques de la présence du sujet dans l'énoncé oral}

Nous allons maintenant examiner la relation, telle qu'elle existe en oralité, entre le sujet réel de l'énonciation (l'interprète, qui peut aussi être éventuellement l'auteur dans quelques cas) et les marques textuelles de la présence dans l'énoncé d'un sujeténonciateur qui ne coïncide pas forcément totalement avec le premier.

Nous avons vu qu'en littérature, il y avait souvent une distance importante entre les deux, introduite par la fiction. Puisque dans la culture orale, la règle semble être celle de l'effacement de l'individu au profit de la communauté, on pourrait s'attendre à ce que ces marques textuelles soient absentes et que notamment le sujet de l'énonciation ne se mette pas en scène dans ses textes, ignorant le recours à la première personne.

Pourtant, nous avons déjà évoqué le cas des conteurs qui, par exemple à la fin des contes, ont parfois recours au «je " pour justifier de façon ludique leur légitimité à narrer ce récit particulier dont ils se présentent comme un personnage-témoin, entrant ainsi par un artifice dans l'univers de la fiction. Il est vrai que dans ce cas, il s'agit d'un « je » très conventionnel qui n’a pas grand-chose à voir avec le sujet-énonciateur réel.

51 Mais dans certaines sociétés de culture orale, il arrive qu'un «je » textuellement présent ait une dimension autobiographique plus prononcée, même si souvent cette référence autobiographique reste très conventionnellement codée. Ainsi, pour revenir sur le cas de l'oralité africaine, il est courant que dans l'épopée peule, wolof, mandingue, avant d'entrer dans son histoire, le griot commence par se présenter et par dire d'où il tient son récit. Le « je » textuel coïncide certes en l'occurrence avec le « je » 
de la réalité de l'énonciation; toutefois il s'agit d'un «moi » social plus que d'un « moi » individuel. La présence de cette personne au seuil de l'énoncé n'est pas destinée à signaler la singularité d'une expression personnelle, mais bien plutôt à justifier l'énonciation par l'appartenance du sujet à un statut social qui le qualifie pour ce faire.

Il peut arriver cependant que la présence textuelle du « moi », dans certaines oeuvres à caractère lyrique, signifie bien l'expression d'une subjectivité individuelle, du moins de la part du sujet créateur qui a inventé l'œuvre et, l'assumant à la première personne, y a mis des éléments autobiographiques. Pour rester en Afrique, cela se rencontre par exemple chez les poètes nzakara dont nous avons déjà parlé, certains d'entre eux, comme Lamadani (de Dampierre, 1987), affectionnant plus que d'autres cette voie du lyrisme personnel. De la part du créateur de l'œuvre, il s'agit bien alors d'une inscription visible du sujet dans l'énoncé même. Mais il ne faut pas oublier qu'en oralité, les oeuvres ainsi créées ont des chances d'être reprises par d'autres interprètes. Dans la mesure où ceux-ci vont conserver l'énonciation à la première personne, on peut alors légitimement se demander comment il faut interpréter ce «je » qui intervient en second lieu et qui n'a pas forcément vécu ce dont parlait, lors de la performance initiale, le « je » de l'inventeur.

Parfois, le « je » engagé de la création initiale peut devenir un « je » plus conventionnel qui ne parle pas d'expérience, mais qui exprime, sous une forme individuelle une pensée ou un état qui correspond à la philosophie ou à la sensibilité de toute la communauté ou du moins d'une de ses fractions constituées. L'œuvre s'est alors greffée au patrimoine parce que l'expérience singulière d'un sujet est devenue emblématique de celle d'un groupe. Dans la culture orale africaine, le « je » n'a de vraies chances de survie que lorsqu'il exprime l'expérience d'un sujet relié à un ensemble.

On s'aperçoit d'ailleurs que parfois des interprètes trouvent eux- mêmes le moyen, en reprenant une œuvre traditionnelle dans un répertoire antérieur, de se réapproprier individuellement ces marques textuelles du sujet, en les réactualisant à leur profit. Un bon exemple en est donné avec la cérémonie du kurubi chez les Dioula de Kong, une société du nord-est de la Côte d'Ivoire. Il est d'usage qu'à cette occasion la femme mariée, dans une sorte de rituel cathartique, exprime ses griefs à l'égard de son entourage. Elle peut, pour cela, soit inventer un chant original qui devra plus ou moins évoquer métaphoriquement sa situation de présumée victime, soit choisir dans le répertoire un chant qui paraisse adapté à cette situation. Comme les récriminations sont toujours plus ou moins les mêmes, mari brutal, inattentif ou injuste, coépouse jalouse, ce n'est pas très difficile de puiser dans le stock existant. Dans ce cas, l'interprète qui s'exprime à la première personne réassume personnellement le chant créé par une autre, en l'appliquant à elle- même. Le texte conserve alors sa valeur de circonstance originelle et par conséquent sa subjectivité individuelle. Cette fois encore, cela n'est possible que dans la mesure où cette succession de subjectivités correspond à une expérience partagée par la communauté des femmes.

Certaines œuvres peuvent ainsi apparaitre conçues pour être régulièrement réinvesties par des sujets qui pourront marquer leur individualité par quelques signes propres: introduction de leur nom, repères topographiques relatifs à leur lieu de résidence. On en a une bonne illustration avec un chant de chasseurs provenant lui aussi de chez les Dioula. Ce chant s'exécute traditionnellement aux funérailles d'un grand chasseur et il est interprété, lorsque la chose est possible, par le fils du défunt. Le canevas en est le suivant : le fils, à la chasse dans la brousse, rencontre successivement des animaux en 
pleurs qui lui apprennent la mort de son père dont ils louent le talent de chasseur. L'homme rentre au village où la nouvelle lui est confirmée et il se lamente alors de cette irréparable perte. À chaque fois qu'un interprète chante rituellement cette œuvre à des funérailles, il est d'usage que, tout en respectant scrupuleusement la structure du récit, il précise dans le chant le nom réel de son père et éventuellement le sien propre qui figurera par exemple dans le texte lorsque les animaux s'adresseront à lui, etc. ${ }^{4}$ La grande différence entre le sujet textuel de l'énonciation tel qu'il se présente d'une part dans la production littéraire orale et d'autre part dans la production littéraire écrite, c'est que le premier est toujours, au moins en apparence, plus conforme à la réalité de l'énonciateur. Cela se comprend dans la mesure où, par principe, l'oralité première est un mode de communication direct et immédiat qui, à la différence du cas de la littérature écrite, impose au consommateur du discours la présence physique de celui qui l'énonce.

En littérature, rien n'empêche un auteur d'âge mûr et de sexe masculin d'écrire une fiction à la première personne où le narrateur se présente comme une belle jeune fille et vice versa. Cela ne gêne guère le lecteur puisque l'auteur réel n'est pas présent de façon sensible au moment où il prend connaissance du texte. Une telle supercherie n'est guère envisageable dans une communication immédiate où l'énonciateur ne peut être que lui- même. Bien sûr, au cours d'un conte par exemple, il peut incarner différents personnages dans le cadre de sa narration, mais dans ce cas, l'auditoire comprend qu'il «joue» des personnages dans le cadre d'une convention dramatique, rôle qu'il ne peut endosser au titre de narrateur principal.

Si le sujet textuel de l'énonciation s'affirme comme l'énonciateur apparent, lorsqu'il se met en scène dans un énoncé de littérature orale, il ne faut cependant pas être dupe de cette présence : ce sujet individuel ne montre au bout du compte de lui que la part conventionnelle que la communauté l'autorise à livrer. Le lyrisme personnel est finalement canalisé et même lorsqu'il multiplie les marques de personnalisation dans son énoncé, l'interprète ne dispose que d'une marge très étroite pour exprimer sa singularité. Il n'est conçu comme sujet individuel que dans le cadre de sa relation à une totalité.

Pour conclure sur ce point, on ne saurait donc penser que le rôle du sujet est réduit à zéro dans l'énonciation de la littérature orale. Qu'il crée ou qu'il répète, cas beaucoup plus fréquent, il agit toujours d'une façon ou d'une autre sur son énoncé. Mais il est vrai cependant que sa marque est beaucoup plus atténuée que dans le cas de la littérature écrite. Ce sujet- énonciateur intervient d'abord comme élément d'un ensemble social plutôt que comme individu prenant ses distances avec le groupe. D'une certaine manière, on peut considérer que, par rapport au phénomène de la création, la culture orale est en quelque sorte victime d'une illusion inverse de celle de la culture écrite. La civilisation de l'écriture, parce qu'elle peut matériellement conserver, au moyen d'une technique graphique, ses productions verbales, ne craint pas la perte de son patrimoine en la matière. C'est pourquoi elle valorise la création originale et peut tomber dans l'illusion que l'auteur d'une oeuvre en est la source unique, sans toujours percevoir les déterminismes socioculturels qui ont accompagné la genèse de cette œuvre.

À l'opposé, la civilisation de l'oralité entretient l'illusion, pour continuer à croire en son identité, que les discours de son patrimoine se transmettent selon une mimesis idéale, alors qu'en réalité l'interprète ne s'efface jamais totalement et s'inscrit nécessairement dans son énoncé. 


\section{BIBLIOGRAPHIE}

BAUMGARDT, Ursula, 2000, Une conteuse peule et son répertoire. Goggo Addi de Garoua (Nord Cameroun),

Paris, Karthala.

DAMPIERRE, Éric de, 1963, Poètes nzakara, Paris, Classiques africains.

DAMPIERRE, Éric de, 1987, Satires de Lamadani, Paris, Classiques africains.

DERIVE, Jean, 1975, Collecte et traduction des littératures orales, Paris, SELAF.

DERIVE, Jean, DUMESTRE, Gérard, 1999, Des hommes et des bêtes, chants de chasseurs mandingues, Paris, Classiques africains.

FINNEGAN, Ruth, 1967, Limba Stories and Story telling, Oxford, Clarendon Press.

JAKOBSON, Roman, BogATYRev, Petr, 1973, Le folklore, forme spécifique de création, Questions de poétique, Paris, Seuil, p. 59-72.

Koudjo, Bienvenu, 1989, La Chanson populaire dans les cultures fon et gun du Bénin, Thèse pour le Doctorat d'État, université de Paris XII.

LUNEAU, René, 1974, Les Chemins de la noce. La femme et le mariage dans la société rurale au Mali, Thèse pour le Doctorat d'État, Université de Paris V.

NORA, Pierre, 1984-92, Les Lieux de mémoire, Paris, Gallimard (Bibliothèque illustrée des histoires), 3 tomes : t. 1 La République (1 vol., 1984), t. 2 La Nation (3 vol., 1986), t. 3 Les France (3 vol., 1992).

ONG, Walter J., 1982, Orality and Literacy, the Technologizing of the Word, Londres, New York, Methuen.

SEYDOU, Christiane, 1991, Bergers des mots, Paris, Classiques africains.

ZUMTHOR, Paul, 1983, Introduction à la poésie orale, Paris, Le Seuil.

\section{NOTES}

1. Le régime d'oralité dite "première » est celui où les énoncés patrimoniaux produits le sont sans aucun support autre que celui de la voix. Ces énoncés n'ont donc pas d'existence en dehors d'une performance donnée. Sur ce concept et la distinction à faire entre oralité première, oralité seconde, néo-oralité, voir, entre autres, Ong (1982) et Zumthor (1983).

2. Voir par exemple, en beaucoup de contes européens se terminant par un mariage, des formules plus ou moins ritualisées comme celle-ci : «j'ai voulu aller à la noce, mais on n'a point voulu de moi parce que je n'avais point de souliers».

3. Voir note 1.

4. On trouvera un chant de ce type dans Derive et Dumestre (1999). 


\section{RÉSUMÉS}

L'article porte sur les modalités de l'énonciation littéraire en situation de culture orale. Il comprend deux temps. Tout d'abord, l'étude des contraintes spatio- temporelles imposées par la communication orale qui, à la différence de la littérature écrite, exige la présence hic et nunc des interlocuteurs. Il se focalise sur les contraintes spatiales, envisageant à la fois la logique des lieux de production et de la mise en espace de la performance en fonction de la nature des discours propres aux différents genres littéraires. En second lieu est étudiée la question du sujet de l'énonciation qui se pose de façon particulière étant donné que l'interprète n'est pas censé, le plus souvent, être l'inventeur, mais le transmetteur du discours qu'il profère. Cette situation suppose des stratégies spécifiques d'inscription du sujet, tant au niveau de l'énonciation qu'à celui de l'énoncé qui sont successivement analysées.

The article analyzes the different modalities of literary performance in oral culture. It is divided into two parts. It first analyzes the spatial-temporal constraints imposed by oral communication which, differently from written literature, requires the presence hic et nunc of the interlocutors. It focuses on spatial constraints by examining the logic of the spaces of production and the spatial configuration of performance based on the nature of the discourse of different literary genres. Second, the subject of the performance is of particular note because the interpreter is not supposed to invent but rather transmit the discourse concerned. The different strategies used are analyzed.

\section{INDEX}

Mots-clés : énonciation, énoncé, espace, performance, sujet, actualisation

Keywords : Performance, Utterance, Space, Subject, Actualization

Thèmes : littérature orale 